\title{
PAPER
}

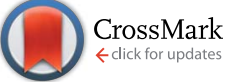

Cite this: RSC Adv., 2016, 6, 25780
Received 27th January 2016 Accepted 2nd March 2016

DOI: $10.1039 / c 6 r a 02479 c$

www.rsc.org/advances

\section{Functionalization of silica through thiol-yne radical chemistry: a catalytic system based on gold nanoparticles supported on amino-sulfide- branched silica†}

\author{
Barbara Ballarin, ${ }^{a}$ Davide Barreca, ${ }^{\mathrm{b}}$ Elisa Boanini, ${ }^{\mathrm{C}}$ Elisa Bonansegna, ${ }^{\mathrm{a}}$ \\ Maria Cristina Cassani, ${ }^{\text {*a }}$ Giorgio Carraro, ${ }^{d}$ Silvia Fazzini, ${ }^{a}$ Adriana Mignani, ${ }^{a}$ \\ Daniele Nanni ${ }^{* a}$ and Davide Pinellie
}

\begin{abstract}
This work proposes a preparation route to heterogeneous catalysts based on gold nanoparticles ( $A u_{N P s}$ ) supported on chemically modified silica. More specifically, the latter is functionalized with amino-sulfide branches (Au-SiO 2 (AeThio) through a thiol-yne radical coupling performed between cysteamine hydrochloride and an alkynyl-substituted triethoxysilane, followed by co-condensation with tetraethoxysilane (TEOS). The target procedure, involving only a gold precursor without any need of additional reducing and/or stabilizing agents, is straightforward, controllable, reproducible, and particularly appealing from a "green" point of view. The supported Au NPs, with an average diameter of $10 \mathrm{~nm}$, possess a remarkable catalytic activity (specific rate constants of the order of $10^{-2} \mathrm{~s}^{-1} \mathrm{mg}_{\mathrm{cat}}{ }^{-1}$ ) in the reduction of 4-nitrophenol (4-NP) to 4-aminophenol (4-AP) by sodium borohydride $\left(\mathrm{NaBH}_{4}\right)$ in aqueous media. The higher performances with respect to previous literature work, along with the possibility of successfully recycling the catalyst, shows the developed materials as attractive functional platforms.
\end{abstract}

\section{Introduction}

In a recent work, we have reported a novel, convenient method for the preparation of gold nanoparticles $\left(\mathrm{Au}_{\mathrm{NPs}}\right)$ supported on nanosilica. Specifically, silica nanoparticles functionalized with alkynyl carbamate moieties $\left(\mathbf{S i O}_{2} @ Y\right.$ Yne, Fig. 1) were synthetized by co-condensation of the di-functional organosilane [3-(2-propynylcarbamate)propyl]triethoxysilane (PPTEOS) with tetraethoxysilane (TEOS) in alkaline medium. ${ }^{\mathbf{1}}$

The alkynyl-carbamate moieties present on the silica nanoparticles were able to capture the gold precursor $\mathrm{HAuCl}_{4}$ and to

${ }^{a}$ Department of Industrial Chemistry "Toso Montanari", University of Bologna, Viale del Risorgimento 4, I-40136 Bologna, Italy. E-mail: maria.cassani@unibo.it; daniele. nanni@unibo.it; Fax: +39051 2093690; +39051 2093654; Tel: +39051 2093700; +390512093623

${ }^{b}$ CNR-IENI c/o Department of Chemical Sciences, University of Padova, Via Marzolo 1, I-35131 Padova, Italy

"Department of Chemistry "Giacomo Ciamician", University of Bologna, Via Selmi 2, I40126 Bologna, Italy

${ }^{d}$ Department of Chemical Sciences, University of Padova and INSTM, Via Marzolo 1, I35131 Padova, Italy

${ }^{e}$ Department of Civil, Chemical, Environmental, and Materials Engineering, University of Bologna, Via Terracini 28, I-40131 Bologna, Italy

$\dagger$ Electronic supplementary information (ESI) available: Additional chemico-physical characterization data for both $\mathbf{S i O}_{2} @ \mathbf{A e T h i o}$ and $\mathbf{A u}-\mathbf{S i O}_{2} @$ AeThio; optical absorption spectra for the reduction of 4-NP to 4-AP; additional catalytic data. See DOI: 10.1039/c6ra02479c

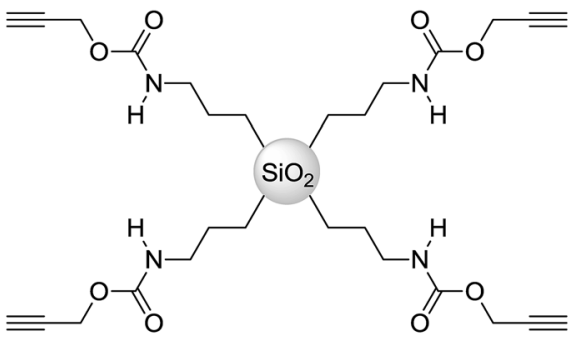

$\mathrm{SiO}_{2} @ Y n e$

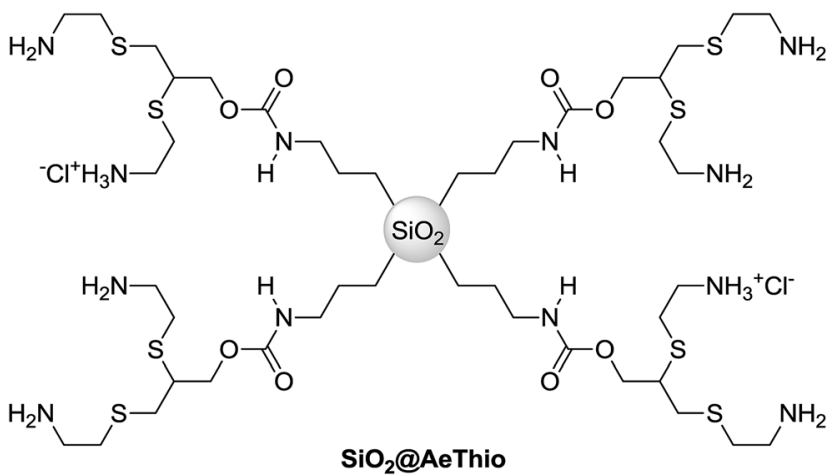

Fig. 1 Alkyne- and amino-sulfide-modified silica nanoparticles $\mathrm{SiO}_{2} \mathrm{aYne}$ and $\mathrm{SiO}_{2} @$ aAeThio. ${ }^{2}$ 


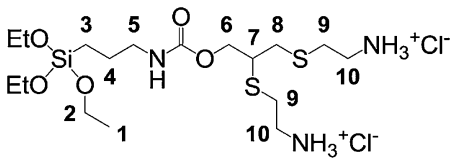

Fig. 2 Compound 1 with NMR numbering scheme.

reduce it, yielding supported $\mathrm{Au}_{\mathrm{NPs}}$. The $\mathbf{A u}-\mathbf{S i O}_{2} @ Y$ Yne systems showed very high activities for the reduction of 4-nitrophenol (4-NP) to 4-aminophenol (4-AP).

An important aspect of these systems is that the presence of the alkyne moiety, either in the starting di-functional organosilane or in the eventual $\mathbf{S i O}_{2} @ Y$ (a) catalyst, can easily allow for further functionalization, for example by a "click-chemistry" approach, yielding a wide variety of novel materials with tuneable chemico-physical properties and performances. Among the most popular "click" reactions, it is worth mentioning the $\mathrm{Cu}$-catalyzed azide-alkyne cycloaddition (CuAAC), ${ }^{3}$ but also very old radical reactions, such as addition of thiols to alkenes and alkynes. The latter processes are of particular relevance, since they can be conveniently used not only for material derivatization, but also in those areas where biotoxicity could be an important issue, i.e. in bioconjugation. ${ }^{4}$ In addition, at variance with CuAAC coupling, such reactions do not require any potentially toxic metal catalyst.

In recent years, radical thiol-yne coupling (TYC, the thermally or photochemically induced radical addition of thiols to alkynes $)^{5}$ has recently emerged as one of the most attractive "click" chemistry procedures. ${ }^{6}$ In particular, the use of TYC in the field of gold nanoparticles is rather unexplored and, to the best of our knowledge, it has been dealt with only in two previous literature works. The former ${ }^{7}$ describes grafting of polymeric monolytes with alkyne groups and their subsequent functionalization with primary-amine- and sulfide-moiety containing branches, capable of anchoring pre-formed gold nanoparticles. The latter ${ }^{8}$ reports the synthesis of a microporous organic polymer by TYC reaction of an aromatic trithiol with an alkynyladamantane. The resulting material is capable of trapping $\mathrm{Au}_{\mathrm{NPs}}$ formed by in situ reduction of $\mathrm{HAuCl}_{4}$ by $\mathrm{NaBH}_{4}$. It is worthwhile noticing that both processes require additional reagents in combination with the support, either to obtain preformed $\mathrm{Au}_{\mathrm{NPs}}{ }^{7}$ or to drive the in situ reduction of suitable $\mathrm{Au}$ precursors. ${ }^{8}$ So far, no related studies involving silica-based systems were ever described in the literature.

The idea of carrying out radical TYC reactions to further functionalize our alkynyl-substituted silica stems from the inherent advantages of thiol-yne chemistry, i.e. the possibility of carrying out TYC-TEC sequences ${ }^{6}$ leading to bis-alkylsulfides instead of mono-vinylsulfides. This feature of thiol-yne chemistry has fuelled a great deal of studies, since it enables the fabrication of highly functionalized materials (e.g. dendrimers) through a single synthetic procedure. In the present case, application of TYC chemistry to $\mathbf{S i O}_{2}$ @Yne would result in a double functionalization of silica with amino-sulfide branches that, thanks to both the amino and the sulfide moieties, could take an active role in complexing the gold precursor $\left(\mathrm{HAuCl}_{4}\right)$, reducing it, and stabilizing the resulting $\mathrm{Au}_{\mathrm{NPs}}$ without the need of any additional reagent.

Basing on the above observations, in this work we present the synthesis of silica functionalized with amino-sulfide branches by radical thiol-yne coupling ( $\mathbf{S i O}_{2} @ A$ AThio, Fig. 1) and the successive preparation of silica-supported $\mathrm{Au}_{\mathrm{NPs}}, \mathbf{A u}-\mathbf{S i O}_{2} @ A$ AThio. The latter system is an efficient heterogeneous catalyst for the reduction of 4-NP, ${ }^{9,10}$ to 4-AP in aqueous media with $\mathrm{NaBH}_{4}$, with specific kinetic constants of the order of $10^{-2} \mathrm{~s}^{-1} \mathrm{mg}_{\text {cat }}{ }^{-1}$.

\section{Experimental section}

\subsection{Materials and methods}

Tetraethoxysilane (TEOS) (99\%), cysteamine hydrochloride (98\%), 2-dimethoxy-2-phenylacetophenone (DMPA), triethylamine (TEA, 99\%), sodium borohydride (98\%), 4-nitrophenol (4-NP), ethanol (99\%), dichloromethane (DCM, 99\%), and hydrochloric acid $(\mathrm{HCl}, 37 \mathrm{wt} \%)$ were purchased from Aldrich ${ }^{\circledR}$. Ethanol was boiled and stored under nitrogen prior to use, whereas the other reagents were used as received. Ammonia solution (30 wt\%, $\left.d=0.904 \mathrm{~g} \mathrm{~mL}^{-1}\right)$ and nitric acid $\left(\mathrm{HNO}_{3}\right.$, $65 \mathrm{wt} \%)$ were purchased from Carlo Erba®. Ultrapure water purified with the Milli-Q plus system (Millipore Co, resistivity over $18 \mathrm{M} \Omega \mathrm{cm}$ ) was used in all cases. $\mathrm{HAuCl}_{4} \cdot 3 \mathrm{H}_{2} \mathrm{O}$, [3-(2-propynylcarbamate)propyl]triethoxysilane (PPTEOS), and the alkyne-modified silica nanoparticles (SiO $\mathbf{S}_{\mathbf{2}} @ \mathbf{Y n e )}$ were prepared as previously reported. ${ }^{\mathbf{1}}$ All glassware was cleaned before use by immersion in aqua regia for several hours, rinsed with ultrapure water and acetone, and dried in an oven. The reactions were monitored by thin-layer chromatography (TLC) on highly purified silica on TLC aluminium foils (w/UV indicator).

\subsection{Material characterization}

NMR spectra were recorded at $298 \mathrm{~K}$ using Varian Inova $\left({ }^{1} \mathrm{H}, 300.1 ;{ }^{13} \mathrm{C}, 75.5 \mathrm{MHz}\right)$, Varian Mercury VX $\left({ }^{1} \mathrm{H}, 399.9 ;{ }^{13} \mathrm{C}\right.$, 100.6 MHz), and Varian Inova $\left({ }^{1} \mathrm{H}, 599.7 ;{ }^{13} \mathrm{C}, 150.8 \mathrm{MHz}\right)$ spectrometers; chemical shifts were referenced internally to residual solvent. ${ }^{11}$ Wherever necessary, detailed ${ }^{1} \mathrm{H}$ and ${ }^{13} \mathrm{C}$ NMR assignments were carried out by gCOSY and gHSQC experiments using standard varian pulse sequences. ESI-MS analyses were performed using a WATERS ZQ 4000 mass spectrometer by direct injection of methanol solutions. ATR-IR spectra (32 scans; resolution $=0.5 \mathrm{~cm}^{-1}$ ) were recorded with a Perkin Elmer spectrum two spectrometer, equipped with an Universal ATR accessory. Thermogravimetric analyses (TGA) were carried out using a Perkin Elmer TGA-7. Heating was performed in a platinum crucible in air flow $\left(20 \mathrm{~cm}^{3} \mathrm{~min}^{-1}\right)$ at a rate of $10{ }^{\circ} \mathrm{C} \mathrm{min}^{-1}$ up to $900{ }^{\circ} \mathrm{C}$. The samples weights were in the range $5-10 \mathrm{mg}$.

X-Ray Photoelectron Spectroscopy (XPS) spectra were recorded by means of a Perkin Elmer $\Phi$ 5600ci spectrometer at a working pressure lower than $10^{-8} \mathrm{mbar}$, using a non-monochromatized AlK $\alpha$ excitation source $(h \nu=1486.6 \mathrm{eV})$. The samples were introduced directly into the analysis chamber by a fast entry lock system. Binding Energy (BE) shifts were corrected for charging by assigning to the $\mathrm{C}$ 1s line of adventitious carbon a value of 
$284.8 \mathrm{eV} .^{12}$ The estimated standard deviation for BEs was $\pm 0.2 \mathrm{eV}$. After a Shirley-type background subtraction, raw spectra were fitted by adopting Gaussian-Lorentzian peak shapes. The atomic compositions were evaluated using sensitivity factors provided by $\Phi$ V5.4A software. For sulfur quantification, in order to avoid interferences, the S 2s peak (see Fig. S3 and S4, ESI $\dagger$ ) was used instead of the S 2p one, since the latter is located very close to the $\mathrm{Si}$ 2s signal. ${ }^{13}$ After a Shirley-type background substraction, ${ }^{12}$ peak fitting was performed by means of a non-linear least-squares deconvolution, using Gaussian-Lorentzian peak shapes.

Transmission Electron Microscopy (TEM) investigation was carried out by means of a Philips CM 100 instrument operated at $80 \mathrm{kV}$. For sample preparation, a drop of the target suspension was transferred onto holey carbon foils supported on conventional copper micro-grids. The Image ${ }^{\circledR}$ picture analyzer ${ }^{14}$ software was used to estimate the average $\mathrm{Au}_{\mathrm{NPs}}$ dimensions, averaging the measurements over at least 100 data points per sample. Scanning Electron Microscopy (SEM) analyses were carried out using a Phenom desktop-Scanning Electron Microscope, Phenom ProX (Alfatest s.r.l) at a primary beam acceleration voltage of $10 \mathrm{kV}$ and equipped with a Silicon Drift Detector (SDD) thermoelectrically cooled ( $\mathrm{LN}_{2}$ free). Specific surface area and pore volume were measured by a Micromeritics ASAP 2020 instrument. The total amount of gold in the different samples was determined with flame Atomic Absorption Spectroscopy (AAS, Thermo Scientific) in air-acetylene flame with a wavelength of $242.8 \mathrm{~nm}$ and a spectral band-width of $0.5 \mathrm{~nm}$. The analyses were conducted on the solid samples by comparison with the calibration line $(y=0.0381 x-$ 0.0124; $\left.R^{2}=0.9997\right)$. The solids used for AAS measurements and catalytic tests were weighed with a Mettler Toledo AT 21 comparator balance. The standards for the direct method were prepared by dilution to $25 \mathrm{~mL}$ of different amounts of a concentrated $\mathrm{HAuCl}_{4}$ aqueous solution with the addition of $6.5 \mathrm{~mL}$ of $\mathrm{NaOH}$ and $2 \mathrm{~mL}$ of aqua regia. The solid samples ( $c a .8 \mathrm{mg}$ ) were first dissolved at $60{ }^{\circ} \mathrm{C}$ in a $\mathrm{NaOH}$ aqueous solution $(2 \mathrm{M}, 1 \mathrm{~mL})$, then $2 \mathrm{~mL}$ of aqua regia were added. Further $5.5 \mathrm{~mL}$ of $2 \mathrm{M} \mathrm{NaOH}$ were added to dissolve all silica totally and finally it was diluted with water to a volume of $25 \mathrm{~mL}$. Optical absorption spectra were recorded using a Hewlett-Packard 8453 diode array spectrophotometer (path length $=1 \mathrm{~cm}$ ), operating in transmittance mode and at normal incidence.

\subsection{Synthesis of 12-[(2-ammonioethyl)thio]-4,4-diethoxy-9- oxo-3,10-dioxa-14-thia-8-aza-4-silahexadecan-16-aminium (1, Fig. 2)}

In a $100 \mathrm{~mL}$ two-neck round bottom flask under nitrogen, DMPA (0.128 g, $0.5 \mathrm{mmol})$, cysteamine hydrochloride $(0.568 \mathrm{~g}, 5$ mmol), and TEA ( $7 \mu \mathrm{L}, 0.05 \mathrm{mmol})$ were added to a solution of PPTEOS $(0.303 \mathrm{~g}, 1 \mathrm{mmol})$ in $20 \mathrm{~mL}$ of ethanol. The reaction mixture was irradiated with a UV lamp operating at $\lambda=365 \mathrm{~nm}$ for $30 \mathrm{~min}$ under stirring. Subsequently, TLC analysis (diethyl ether/petroleum ether $=1 / 1$ ) showed the complete disappearance of the starting PPTEOS. A small amount of the crude material was taken for the NMR and ESI-MS characterization, whereas the main portion was straightforwardly used for the successive preparation of $\mathbf{S i O}_{2} @ A$ AThio.
${ }^{1} \mathrm{H}$ NMR (599.7 MHz, $\left.\mathrm{D}_{2} \mathrm{O}\right): \delta 4.30\left(\mathrm{dd}, 2 \mathrm{H}^{6},{ }^{3} \mathrm{~J}_{\mathrm{H}, \mathrm{H}}=6.0 \mathrm{~Hz}\right.$, $\left.{ }^{2} J_{\mathrm{H}, \mathrm{H}}=18 \mathrm{~Hz}\right), 3.65\left(\mathrm{q}, 6 \mathrm{H}^{2},{ }^{3} J_{\mathrm{H}, \mathrm{H}}=6.0 \mathrm{~Hz}\right), 3.45\left(\mathrm{~m}, 1 \mathrm{H}^{7}\right), 3.26$ $\left(\mathrm{m}, 4 \mathrm{H}^{10}\right), 3.13\left(\mathrm{t}, 2 \mathrm{H}^{5}\right), 3.01\left(\mathrm{~m}, 2 \mathrm{H}^{8}\right), 2.94\left(\mathrm{~m}, 4 \mathrm{H}^{9}\right), 1.61(\mathrm{~m}$, $\left.2 \mathrm{H}^{4}\right), 1.18\left(\mathrm{t}, 9 \mathrm{H}^{1},{ }^{3} \mathrm{~J}_{\mathrm{H}, \mathrm{H}}=6.0 \mathrm{~Hz}\right), 0.66\left(\mathrm{~m}, 2 \mathrm{H}^{3}\right) .{ }^{13} \mathrm{C} \mathrm{NMR}(150.8$, DMSO-d $\left.{ }_{6}\right): \delta 155.88(\mathrm{C}=\mathrm{O}), 65.84\left(\mathrm{C}(\mathrm{O}) \mathrm{OCH}_{2}\right), 57.49\left(\mathrm{SiOCH}_{2}-\right.$ $\left.\mathrm{CH}_{3}\right), 43.37\left(\mathrm{CH}_{2} \mathrm{NH}\right), 38.75\left(\mathrm{SCH}_{2} \mathrm{CH}_{2} \mathrm{NH}_{3}{ }^{+}\right), 33.83\left(\mathrm{CHCH}_{2} \mathrm{~S}\right)$, $29.35\left(\mathrm{SCH}_{2} \mathrm{CH}_{2} \mathrm{NH}_{3}{ }^{+}\right), 28.16\left(\mathrm{CHCH}_{2} \mathrm{~S}\right), 22.51\left(\mathrm{SiCH}_{2} \mathrm{CH}_{2} \mathrm{CH}_{2}{ }^{-}\right.$ $\mathrm{NH}), 18.63\left(\mathrm{SiOCH}_{2} \mathrm{CH}_{3}\right), 11.47\left(\mathrm{SiCH}_{2} \mathrm{CH}_{2} \mathrm{CH}_{2} \mathrm{NH}\right) . \operatorname{ESI}-\mathrm{MS}(+)$ $(\mathrm{MeOH}, m / z): 458[\mathrm{M}]^{+}$.

\subsection{Synthesis of $\mathrm{SiO}_{2} @ A$ AThio}

TEOS (0.88 mL, $9.4 \mathrm{mmol})$, ethanol $(9.0 \mathrm{~mL})$, and $\mathrm{NH}_{3}(1.89 \mathrm{~mL}$, $16.0 \mathrm{mmol}$ ) were added to the same flask containing 1 . The reaction mixture was stirred at $40{ }^{\circ} \mathrm{C}$ for $24 \mathrm{~h}$ under nitrogen. The obtained $\mathbf{S i O}_{2} @$ AeThio was isolated by centrifugation (4500 rpm for $20 \mathrm{~min}$ ) and re-dispersed in ethanol $(20 \mathrm{~mL})$. This procedure was repeated twice in $\mathrm{EtOH}$ and once in Milli-Q water. Finally, the sample was dried in oven at $40{ }^{\circ} \mathrm{C}$ for $4 \mathrm{~h}$, ultimately yielding a white powder $(0.380 \mathrm{~g})$. The percentage of amino/ammonium groups present on the silica surface was calculated using the following two-step acid-base titration analysis. In a typical analysis, $0.200 \mathrm{~g}$ of $\mathbf{S i O}_{2} @ A$ Thio was placed in a $20 \mathrm{~mL}$ vial and $10 \mathrm{~mL}$ of $0.05 \mathrm{M}$ solution of $\mathrm{HCl}$ were added. After $30 \mathrm{~min}, 3 \mathrm{~mL}$ of the supernatant solution were removed and titrated with $0.05 \mathrm{M} \mathrm{NaOH}$ to a phenolphthalein endpoint. The amine group concentration was calculated based on the $\mathrm{HCl}$ amount consumed by the silica sample. In the second step, the ammonium-containing $\mathbf{S i O}_{2} @ A$ AThio sample, after isolation and thorough washing with absolute ethanol, was placed in a $20 \mathrm{~mL}$ vial and allowed to react with $10 \mathrm{~mL}$ of $0.05 \mathrm{M} \mathrm{NaOH}$. After stirring for $1 \mathrm{~h}$, a $3 \mathrm{~mL}$ aliquot of the supernatant solution was removed and titrated with $0.05 \mathrm{M} \mathrm{HCl}$ to a phenolphthalein endpoint. This two-step titration was repeated twice for three different samples.

\subsection{Synthesis of Au-SiO @AeThio}

$60 \mathrm{mg}$ of $\mathrm{SiO}_{2} @$ AeThio and $15 \mathrm{~mL}$ of ultrapure water were introduced in a $50 \mathrm{~mL}$ round bottom flask immersed in a ultrasonic bath. After the complete solid dissolution (10 min), $15 \mathrm{~mL}$ of $1 \mathrm{mM} \mathrm{HAuCl}{ }_{4}$ were added. ${ }^{15}$ The reaction was then carried out at $90{ }^{\circ} \mathrm{C}$ for $1 \mathrm{~h}$ under stirring, with a concomitant progressive change of the initial pink color into red-purple. At the end of the reaction, a small part of the crude material was taken for TEM analyses, whereas the remaining portion was separated by centrifugation (4500 rpm for $30 \mathrm{~min}$ ) and redispersed twice in EtOH and twice in water. The collected

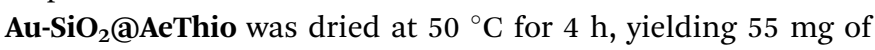
a deep pink powder (Scheme $\mathrm{S} 1 \dagger$ ). The Au content was determined by AAS to be $4.4 \mathrm{wt} \%$.

\subsection{Catalytic tests}

The catalytic activity of $\mathbf{A u}-\mathbf{S i O}_{2} @ A$ AeThio samples was investigated in the reduction of 4-nitrophenol (4-NP) to 4-aminophenol (4-AP) in the presence of an excess of $\mathrm{NaBH}_{4}$ at $25{ }^{\circ} \mathrm{C}$. The reaction was monitored by UV-vis spectroscopy (further details are reported in the ESI $\dagger$ ). 


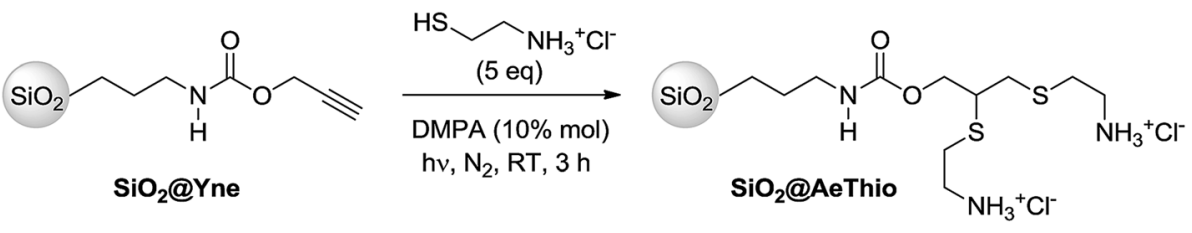

Scheme 1 Synthesis of $\mathrm{SiO}_{2}\left(\mathrm{aAeThio}\right.$ by functionalization of $\mathrm{SiO}_{2} \mathrm{aYne}$.

\section{Results and discussion}

\subsection{Synthesis of $\mathrm{SiO}_{2}$ @AeThio}

We first tried to modify the previously reported alkyneterminated $\mathbf{S i O}_{2} @ Y n e$ by post-functionalization with cysteamine hydrochloride to obtain aminosulfide branches by using the radical TYC reaction. As depicted in Scheme 1, the click reaction on $\mathbf{S i O}_{2}$ @Yne was performed in the presence of an excess of cysteamine hydrochloride and DMPA as photoinitiator (10\% mol with respect to cysteamine hydrochloride) under a nitrogen atmosphere by exposure to a light with $\lambda=365 \mathrm{~nm}$ for $3 \mathrm{~h}$ at room temperature.

The recovered light yellow powder was then thoroughly characterized by means of TGA, FT-IR, Dynamic Light Scattering (DLS), TEM, and XPS analysis. All the collected data indicated that an incomplete thiol-yne reaction had occurred and it was estimated by XPS spectroscopy that ca. $20 \%$ of the alkyne moieties were converted to bis-sulfide adducts. Attempts of improving the conversion by changing the reaction conditions (thiol/alkyne molar ratio, temperature, and solvent) did not lead to any significant improvement. ${ }^{16}$

Based on these results, the synthetic approach was completely changed and we tried to functionalize first the starting PPTEOS, aiming then to co-condensate the resulting branched silane with TEOS. The TYC reaction of PPTEOS was carried out under the conditions described in Scheme 2. In this case, a rewarding complete, selective conversion of PPTEOS to the bis-sulfide adduct 12-[(2-ammonioethyl)thio]-4,4-diethoxy9-oxo-3,10-dioxa-14-thia-8-aza-4-silahexadecane-16-aminium, (1), was successfully obtained.

As a matter of fact, the reaction afforded mixtures of bis- (1) and mono-adducts (2, Fig. 3) depending on the experimental conditions. Upon raising the cysteamine/PPTEOS molar ratio, a parallel increase of the $1: 2$ ratio was observed, as evaluated by NMR analyses of the crude material. Upon using a cysteamine/PPTEOS molar ratio equal to 5 , the $1: 2$ ratio was $c a$. 8 . Only in the presence of catalytic amounts of TEA, the 2 monoadducts were absent in the final reaction mixture. By means of TLC cromatography, vinil-sulfides 2 ( $E / Z$ mixture) could be isolated in sufficient amounts for ${ }^{1} \mathrm{H}$ NMR analysis (Fig. S1 $\dagger$ ). Since 1 could not be isolated by preparative column chromatography, due to its complete hydrolysis, the bis-sulfide moiety was introduced into the $\mathrm{SiO}_{2}$ network by a one-pot cocondensation of crude 1 and TEOS in basic medium at $40{ }^{\circ} \mathrm{C}$ to give $\mathbf{S i O}_{2} @$ AeThio (Scheme 2).

The chemical composition of $\mathbf{S i O}_{2} @ A$ AThio was analyzed by Attenuated Total Reflectance IR (ATR-FT-IR) and XPS spectroscopy. In the ATR-FT-IR spectrum, the main bands at 1700 and $1539 \mathrm{~cm}^{-1}$ are respectively assigned to the $\nu_{\mathrm{s}}(\mathrm{C}=\mathrm{O})$ and $\delta(\mathrm{NH})$ vibrations of the carbamic moiety ${ }^{\mathbf{1 7}}$ (Fig. S2 $\uparrow$ ), suggesting the successful incorporation of 1 into the $\mathrm{SiO}_{2}$ network. XPS analyses further confirmed that the target functional groups were

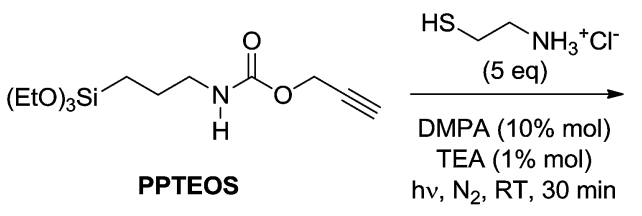<smiles>CCO[SiH2]CCCNC(=O)OCC(CSCC[NH3+])SCC[NH3+]</smiles>

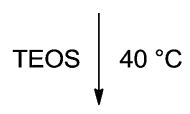

$\mathrm{SiO}_{2} @ A$ AeThio

Scheme 2 Synthesis of $\mathrm{SiO}_{2}$ aAeThio by functionalization of PPTEOS followed by co-condensation with TEOS.<smiles>CCO[SiH2]CCCNC(=O)OC/C=C/SCC[NH3+]</smiles>

(E)-2<smiles>CCO[SiH2]CCCNC(=O)OC/C=C\SCC[NH3+]</smiles>

(Z)-2

Fig. 3 Mono-adducts derived from thiol-yne coupling between PPTEOS and cysteamine. 
effectively anchored to the silica surface. The survey spectrum (Fig. 4a) was dominated by the presence of C, O, Si, S, N as the major elements, as expected. The $\mathrm{C} 1 \mathrm{~s}$ signal (Fig. $4 \mathrm{~b}$ ) could be fitted by means of three contributing bands, centered at 284.8 (I; $67.4 \%$ of the total C signal), 286.3 (II; $24.6 \%$ of the total C signal) and $289.1 \mathrm{eV}$ (III; $8.0 \%$ of the total signal), which were attributed to $\mathrm{C}-\mathrm{C}, \mathrm{C}-\mathrm{H}$, and/or $\mathrm{C}-\mathrm{Si}$; $\mathrm{C}-\mathrm{N}$ and/or $\mathrm{C}-\mathrm{O}$; and $\mathrm{O}-\mathrm{C}=\mathrm{O}$ bonds, respectively. ${ }^{\mathbf{1 , 1 3}}$ These features confirmed the silica functionalization according to the scheme proposed in Fig. 1b ( $\mathbf{S i O}_{2} @ A$ AeThio). It is worthwhile noticing that the first band also contains contributions from adventitious carbon, whose presence is ubiquitous on air-exposed specimens. The fitting of the $\mathrm{N}$ 1s signal (Fig. 4c) evidenced the presence of two
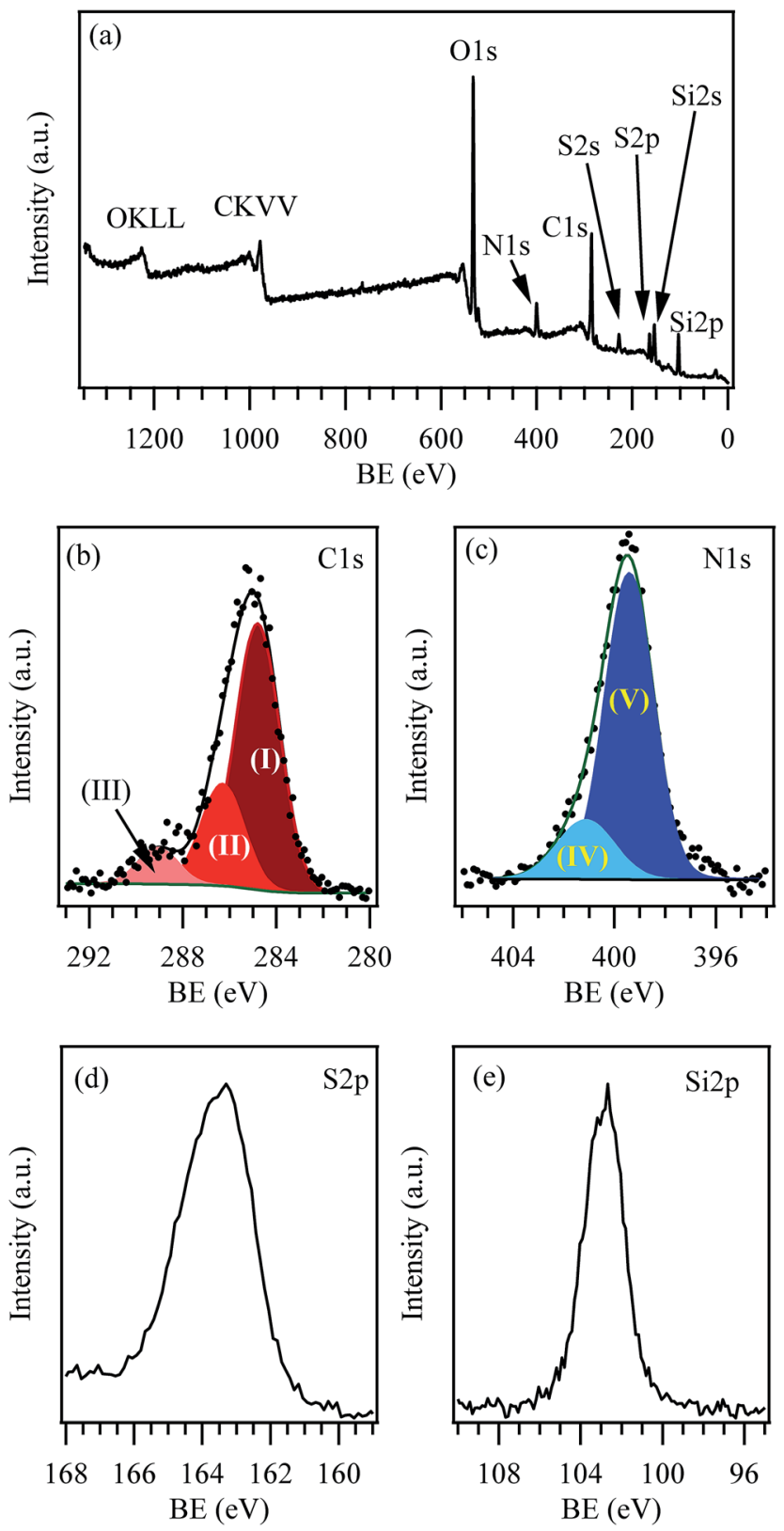

Fig. 4 (a) Surface wide-scan XPS spectrum for the $\mathrm{SiO}_{2}(\mathrm{aAeThio}$ sample. The detailed (b) C 1s, (c) N 1s, (d) S 2p, and (e) Si $2 p$ regions are also displayed. contributions. The main one $(\mathrm{V}$, located at $\mathrm{BE}=399.5 \mathrm{eV})$ was in good agreement with the presence of $\mathrm{N}$ in amino-groups. ${ }^{13} \mathrm{In}$ addition, the tailing at higher BEs (second component, IV, BE = $401.1 \mathrm{eV}$ ) could be ascribed to $\mathrm{N}$ in $\mathrm{NH}-\mathrm{COO}$ - groups. ${ }^{18}$ The $\mathrm{S} 2 \mathrm{p}$ (Fig. 4d) and S 2s (Fig. S3b $\dagger$ ) peak positions (BE $=163.4$ and $227.5 \mathrm{eV}$, respectively) suggested the occurrence to $\mathrm{S}$ in thiazol species. $^{13}$

The presence of a silica-like network was confirmed by the analysis of the O 1s (Fig. S3a $\dagger$ ) and Si 2p (Fig. 4e) photoelectron peaks, that were located at average BE values of $532.4 \mathrm{eV}$ and $102.9 \mathrm{eV}$, respectively. ${ }^{13,19}$ In the case of oxygen, the contribution of $\mathrm{O}$ atoms in the ligand chains could not be resolved with respect to that of $\mathrm{SiO}_{2}$. The calculated $\mathrm{O} / \mathrm{Si}$ atomic ratio (2.9), higher than the theoretical one expected for $\mathrm{SiO}_{2}$, could be traced back both to the surface functionalization and, mostly, to air exposure and consequent water adsorption, as already reported for similar systems. ${ }^{1}$ In fact, $-\mathrm{OH}$ contributions give typically raise to a signal with BEs very close to the one of lattice oxygen in $\mathrm{SiO}_{2}{ }^{19}$

The TGA analyses of $\mathbf{S i O}_{2} @ A$ AThio (Fig. S5 $\dagger$ ) showed three regions of mass loss: 40-160 ${ }^{\circ} \mathrm{C}$ (adsorbed water and ethanol), $160-500{ }^{\circ} \mathrm{C}$ (organic material), and $500-800{ }^{\circ} \mathrm{C}$ (mainly dehydration of Si-OH groups). The weight loss of the organic material was $\approx 16 \%$, in line with the expected organic moieties incorporation. The morphological characterization of $\mathbf{S i O}_{2}$ @AeThio was carried out by means of TEM and SEM analyses. TEM images, collected after samples sonication, showed the presence of compact aggregates of particles, whereas separated particles could never be observed (Fig. S6a $\dagger$ ). SEM micrographs (Fig. S6b $\dagger$ ) and the relative size distribution histogram (Fig. S6c $\dagger$ ) showed a particles size distribution between $180 \mathrm{~nm}$ and $350 \mathrm{~nm}$. The percentage of amino/ammonio groups present on the silica surface, estimated by acid-base titration, yielded $77 \%$ of $-\mathrm{NH}_{2}$ groups $\left(1.22 \mathrm{mmol} \mathrm{g}_{\text {silica }}{ }^{-1}\right)$ and $23 \%$ of $-\mathrm{NH}_{3}{ }^{+}$groups $\left(0.37 \mathrm{mmol} \mathrm{g}_{\text {silica }}{ }^{-1}\right)$. The specific surface area $\left(S_{\mathrm{BET}}\right)$ and pore volume $\left(V_{\mathrm{p}}\right)$ of $\mathbf{S i O}_{2} @ A$ AThio were evaluated by analysis of the nitrogen adsorption/desorption isotherms (Fig. S8 and S9†), yielding values of $6.3 \mathrm{~m}^{2} \mathrm{~g}^{-1}$ and $0.021 \mathrm{~cm}^{3} \mathrm{~g}^{-1}$, respectively. These values were dramatically lower than those observed for the silica-functionalized systems previously prepared by our group,$^{20}$ and indicated the occurrence of a negligible porosity in the target materials.

\subsection{Synthesis of Au-SiO ${ }_{2} @ A e T h i o$}

The silica-supported gold nanoparticles (Au-SiO $\left.\mathbf{A}_{2} @ \mathbf{A e T h i o}\right)$ were prepared by simply adding an aqueous solution of $\mathrm{HAuCl}_{4}$ (1 $\mathrm{mM})$ to a stirred dispersion of $\mathbf{S i O}_{2} @ A$ AThio in water $\left(2 \mathrm{mg} \mathrm{mL}{ }^{-1}\right)$ at $90{ }^{\circ} \mathrm{C}$ for $1 \mathrm{~h}$, as described in the Experimental section. ${ }^{15}$

The crude material, sampled before centrifugation to allow detection of possible non-anchored nanoparticles, was investigated by TEM. The obtained micrographs (Fig. 5) showed that the silica surface was completely decorated with immobilized $\mathrm{Au}_{\mathrm{NPs}}$ having an average diameter of $13 \pm 5 \mathrm{~nm}$. SEM images of

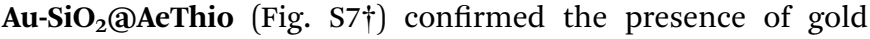



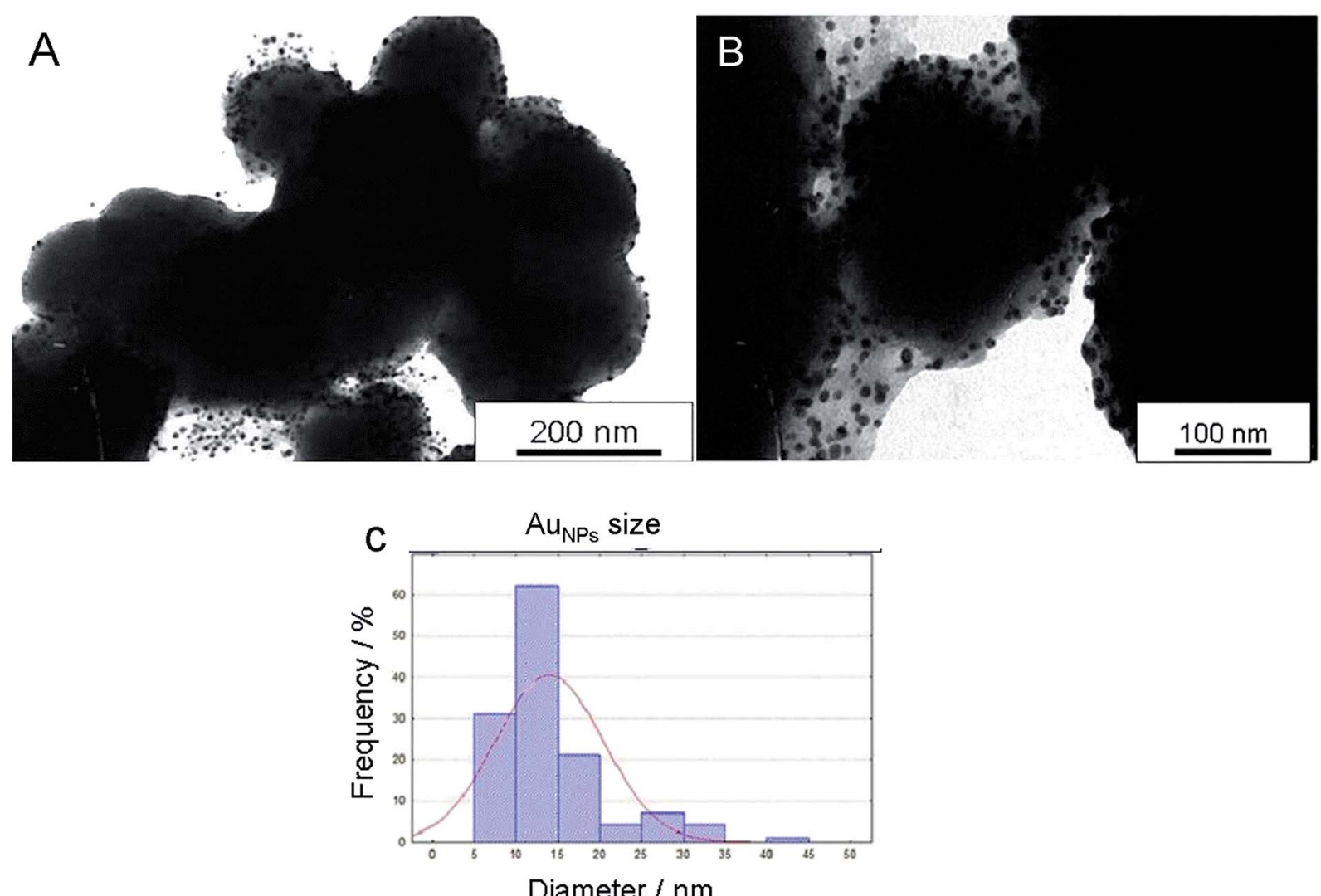

Fig. 5 Representative TEM images at different magnifications (A and B) and average size distributions (C) for supported AunPs in Au$\mathrm{SiO}_{2} @ \mathrm{aAeThio.}$

nanoparticles homogeneously distributed on all functionalized silica surface.

The ATR-FT-IR spectrum of Au-SiO $\mathbf{S}_{2}$ @AeThio (Fig. S2 $\dagger$ ) is substantially identical to that of $\mathbf{S i O}_{2} @ A$ AThio. XPS survey spectra of the Au-SiO $\mathbf{S}_{2}$ @AeThio systems showed features qualitatively similar to those of the gold-free silica specimen (Fig. 6 and $\mathrm{S} 4 \dagger$ ). The $\mathrm{C}$ 1s peak (Fig. 6a) was decomposed into 3 components, centered at $\mathrm{BE}=284.8$ ( $63.6 \%$ of the total $\mathrm{C})$, 286.4 (29.7\% of the total $\mathrm{C})$, and $288.9 \mathrm{eV}$ ( $6.7 \%$ of the total $\mathrm{C})$, in perfect agreement with the $\mathbf{S i O}_{2} @ A$ AeThio system. In a similar way, $\mathrm{N} 1 \mathrm{~s}$ peak was characterized by two bands at $399.7 \mathrm{eV}(\mathrm{V} ; 66.8 \%$ of the total $\mathrm{N})$ and $400.9 \mathrm{eV}(\mathrm{IV} ; 33.2 \%$ of the total N). No significant chemical shifts of the $\mathrm{S} 2 \mathrm{p} / \mathrm{S} 2 \mathrm{~s}$ signals (Fig. $\mathrm{S} 4 \dagger$ ) were detected with respect to the previous case. The last observation holds even in the case of $\mathrm{Si}$ and $\mathrm{O}$ signals (Fig. 6c and S4 $\dagger$ ), suggesting thus that the silica matrix did not undergo any major chemical alteration upon functionalization with gold nanoparticles.

At variance with sample $\mathbf{S i O}_{2} @ A$ AThio, the appearance of an additional signal corresponding to Au $4 \mathrm{f}$ (Fig. S4a† and $6 \mathrm{~d}$ ), with the most intense spin-orbit split component, $\mathrm{Au}$ $4 \mathrm{f}_{7 / 2}$, located at a $\mathrm{BE}=84.2 \mathrm{eV}$, suggested the occurrence of pure metallic Au aggregates. ${ }^{\mathbf{1 2 , 1 3 , 2 1 , 2 2}}$ In line with a recent publication from some of us, ${ }^{\mathbf{1}}$ no signals attributable to oxidized gold species [Au(I) and/or Au(III)] could be clearly detected.

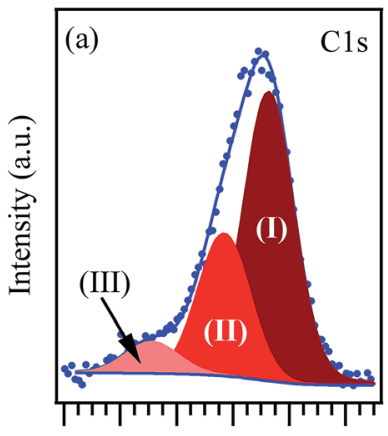

292290288286284282

$\mathrm{BE}(\mathrm{eV})$

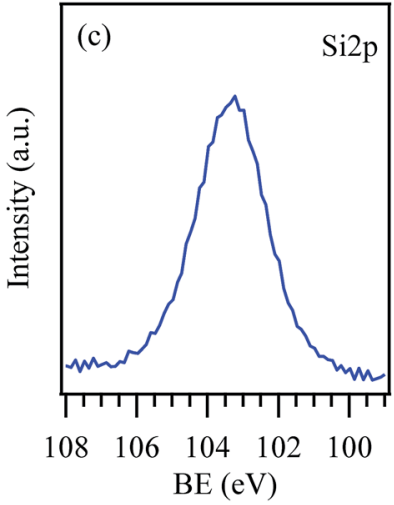

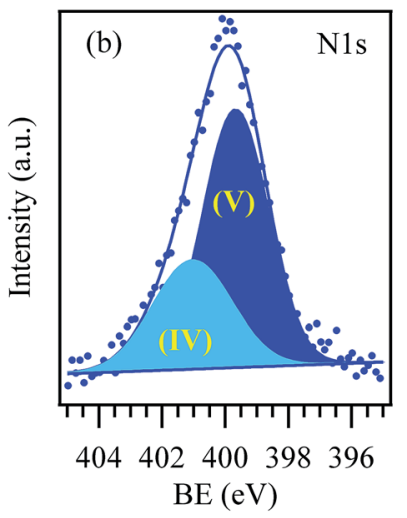

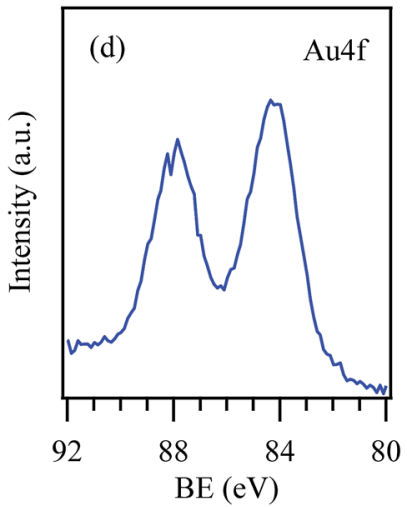

Fig. 6 Surface $C$ 1s (a), N 1s (b), Si 2p (c), and Au 4f (d), and photoelectron signals for $\mathrm{Au}-\mathrm{SiO}_{2} @ \mathrm{AeThio}$ specimen. 


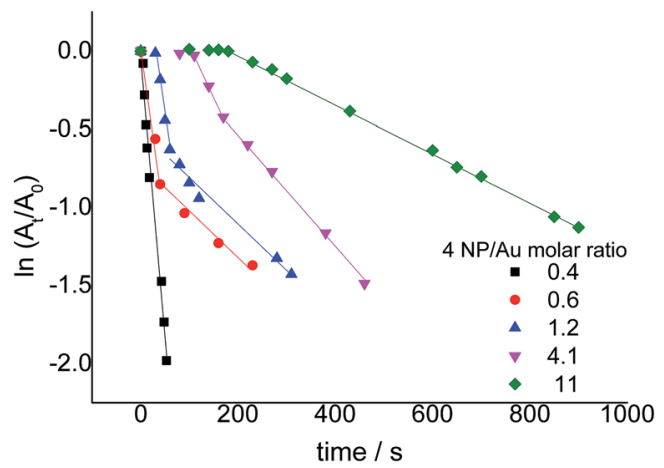

Fig. 7 Plots of $\ln \left(A_{t} / A_{0}\right)$ vs. time for the reduction of 4-NP with Au$\mathrm{SiO}_{2}$ @AeThio using different 4-NP/Au molar ratios. Reaction conditions: aqueous media at $25{ }^{\circ} \mathrm{C}, 4-\mathrm{NP}=0.16 \mu \mathrm{mol}, \mathrm{NaBH}_{4}=0.25$ $\mathrm{mmol}$.

\subsection{Catalytic activity}

The catalytic activity of Au-SiO $\mathbf{S}_{2} @$ AeThio was tested in the reduction of 4-NP to 4-AP, according to what reported in the ESI. $\dagger^{23-28}$ The plots of $\ln \left(A_{t} / A_{0}\right)$ as a function of reaction time $(t)$ using different $4-\mathrm{NP} / \mathrm{Au}$ molar ratios, where $A_{t}$ and $A_{0}$ are the absorbance values at $400 \mathrm{~nm}$ at time $t$ and 0 , respectively, are reported in Fig. 7 . The kinetic constants were calculated as the slope of the linear ranges of these plots, as the ratio of $A_{t} / A_{0}$ is proportional to the ratio of $C_{t} / C_{0}$, where $C_{t}$ is the concentration of $4-\mathrm{NP}$ at the reaction time $t$ and $C_{0}$ is the initial concentration of 4-NP.

As can be observed in Fig. 7, various induction times $\left(t_{\text {in }}\right)$, i.e. a period for catalyst activation, and two different kinetic regimes were detected. A $t_{\text {in }}$ was observed for all considered 4-NP/Au molar ratios except for the lowest ratio 0.4 (see Table 1). This behavior is more evident for the highest 4-NP/Au molar ratio values 4.1 and 11 , i.e., with the lowest catalyst amounts. The $t_{\text {in }}$ period was reduced when 4-NP was added as the last reactant, probably due to a better dispersion of the catalyst owed to the presence of hydrogen bubbles that were produced in the reaction medium by the presence of $\mathrm{NaBH}_{4}$ in water. The results of other catalytic tests carried out varying the addition order of 4-NP, $\mathrm{NaBH}_{4}$, and the catalyst are reported in Table S1. $\dagger$

The kinetic profiles for $4-\mathrm{NP} / \mathrm{Au}$ molar ratios greater than 0.4 showed two linear trends, corresponding to two different kinetic constants. This behavior, actually already reported for other $\mathrm{Au}_{\mathrm{NPs}}$-supported systems, ${ }^{29,30}$ suggested that the main reduction reaction $\left(k_{1}\right)$ was followed at some point by a successive process $\left(k_{2}\right)$ that occurred with a lower reaction rate. Table 1 reports the kinetic constant values for the studied 4-NP/Au molar ratios.

As the change in the reaction rate depends on the available active sites of the catalyst, an increasing catalyst concentration must necessarily result in an increase of the reaction rate. As far as the first slope is concerned, Au-SiO $\mathbf{S}_{2} @$ AeThio showed very high catalytic activity towards reduction of 4-NP, with a kinetic constant $k_{1}$ of $\approx 10^{-2} \mathrm{~s}^{-1}$. These values are one order of magnitude higher than the ones generally obtained for heterogeneous catalysts in identical reductions, ${ }^{26,27}$ and are comparable with those reported in our previous Au-SiO $\mathbf{A}_{2} @ Y$ Yne systems.

The slowing down of the reaction rate observed for $4-\mathrm{NP} / \mathrm{Au}$ molar ratios greater than 0.4 (second slope) could be tentatively ascribed to a by-product adsorption on the catalyst surface, leading, in turn, to a lower number of available catalytic active sites. As a consequence, additional experiments were carried out in the presence of 4-AP already at the beginning of the reaction (see ESI, $\uparrow$ Section 4, for further details). Under these conditions, the same behavior with two kinetic constants was observed for all 4-NP/Au molar ratios, including 0.4. Additionally, as reported in Table 1 , a remarkable decrease of the $k_{1}$ values was observed, hence confirming that the catalyst was indeed deactivated/ poisoned by adsorbed products. Regarding this point, it was possible to observe that, after $\approx 80 \mathrm{~s}$, the bands at $\lambda=260$ and $330 \mathrm{~nm}$, attributable to 4-AP, began to decrease and, simultaneously, a band at $\lambda=230 \mathrm{~nm}$ appeared (compare Fig. S16, S17 and Table $\mathrm{S} 2 \dagger)$. This band might be tentatively assigned to 4,4'-azoxyphenol, ${ }^{31}$ a by-product deriving from a condensation reaction between the reagent $4-\mathrm{NP}$ and the product 4-AP, commonly obtained in reductions of 4-NP with borohydrides. ${ }^{32,33}$ This compound could be the main responsible for catalyst poisoning, accounting thus for the observed behavior. It is worth noting that, intriguingly, this overall occurrence (i.e. both the double kinetic behavior and the appearance of the additional UV band at $230 \mathrm{~nm}$ ) has already been observed ${ }^{\mathbf{3 4 , 3 5}}$ but no authors have ever commented on it, even when the catalytic plots were unquestionably far from linear. ${ }^{35}$ On the contrary, in our opinion, this is a point that cannot be withheld, since it can crucially affect the whole efficiency of the catalytic system.

Basing on these observations, further efforts were dedicated to the possibility of catalyst recycling. In the absence of any treatment, a decrease in the kinetic constant values of about $50 \%$ took place already in the second cycle (Table S3†). In order to reduce the presence of adsorbed molecules (both 4-AP and by-

Table 1 Induction time $\left(t_{\text {in }}\right)$, observed $\left(k_{1,2}\right.$ obs), and specific $\left(k_{1,2}\right)$ kinetic constant values of Au-SiO ${ }_{2} @ A$ AeThio for 4-NP reduction using different 4-NP to Au molar ratios, and the same specific $\left(k_{1,2}\right)$ kinetic constant values in presence of 4 -AP at $t=0$

\begin{tabular}{lllllllll}
\hline $\begin{array}{l}\text { 4-NP/Au } \\
\text { molar ratio }\end{array}$ & $\begin{array}{l}t_{\text {in }} \\
(\mathrm{s})\end{array}$ & $\begin{array}{l}k_{1} \mathrm{obs}^{a} \\
\left(10^{-2} \mathrm{~s}^{-1}\right)\end{array}$ & $\begin{array}{l}k_{2} \mathrm{obs}^{a} \\
\left(10^{-2} \mathrm{~s}^{-1}\right)\end{array}$ & $\begin{array}{l}\text { Cat. weight } \\
(\mathrm{mg})\end{array}$ & $\begin{array}{l}k_{1}^{b, c} \\
\left(10^{-2} \mathrm{~s}^{-1} \mathrm{mg}_{\mathrm{cat}}{ }^{-1}\right)\end{array}$ & $\begin{array}{l}k_{2}{ }^{b, c} \\
\left(10^{-2} \mathrm{~s}^{-1} \mathrm{mg}_{\mathrm{cat}}{ }^{-1}\right)\end{array}$ & $\begin{array}{l}\text { With 4-AP at } t=0 k_{1}^{b, c} \\
\left(10^{-2} \mathrm{~s}^{-1} \mathrm{mg}_{\mathrm{cat}}{ }^{-1}\right)\end{array}$ & $\begin{array}{l}\text { With 4-AP at } t=0 k_{2}^{b, c} \\
\left(10^{-2} \mathrm{~s}^{-1} \mathrm{mg}_{\mathrm{cat}}{ }^{-1}\right)\end{array}$ \\
\hline 0.4 & - & 3.68 & - & 1.80 & $2.0 \pm 0.1$ & - & $0.6 \pm 0.1$ & $0.15 \pm 0.05$ \\
0.6 & 15 & 2.62 & 0.34 & 1.20 & $2.2 \pm 0.1$ & $0.3 \pm 0.1$ & $1.3 \pm 0.1$ & $0.2 \pm 0.1$ \\
1.2 & 38 & 2.10 & 0.44 & 0.60 & $3.5 \pm 0.1$ & $0.7 \pm 0.1$ & $2.8 \pm 0.2$ & $0.5 \pm 0.1$ \\
4.1 & 140 & 0.69 & 0.25 & 0.176 & $3.9 \pm 0.1$ & $1.4 \pm 0.1$ & $1.2 \pm 0.4$ & $0.6 \pm 0.4$ \\
11 & 250 & - & 0.15 & 0.065 & - & $2.3 \pm 0.1$ & - & -
\end{tabular}

${ }^{a}$ Observed $k_{i}=$ slope of the linear range of $\ln \left(A_{t} / A_{0}\right) v s$. time plot. ${ }^{b}$ Specific $k_{i}=$ observed k per mg of catalyst. ${ }^{c}$ Mean of 3 determinations. 
Table 2 Specific kinetic constant values $\left(k_{1} / 10^{-2} \mathrm{~s}^{-1} \mathrm{mg}_{\mathrm{cat}}{ }^{-1}\right)$ using fresh (first cycle) and recycled (second to fifth cycle) Au-SiO $\mathrm{aAeThio}$ catalyst with 4-NP/Au $=0.4$

\begin{tabular}{|c|c|c|c|c|c|}
\hline Au-SiO ${ }_{2} @ A e T h i o$ & 1st cycle & 2nd cycle & 3rd cycle & 4th cycle & 5th cycle \\
\hline$t_{\text {in }}(\mathrm{s})$ & - & - & - & 10 & 10 \\
\hline$k^{a}\left(10^{-2} \mathrm{~s}^{-1} \mathrm{mg}_{\mathrm{cat}}{ }^{-1}\right)$ & $2.1 \pm 0.1$ & $2.2 \pm 0.1$ & $2.1 \pm 0.1$ & $2.2 \pm 0.1$ & $2.2 \pm 0.1$ \\
\hline
\end{tabular}

products) on its surface, after each catalytic cycle the catalyst was separated from the reaction mixture by centrifugation, then extracted with ethyl acetate, dried, and eventually reused in the subsequent cycle. Correspondingly, the catalytic activity of

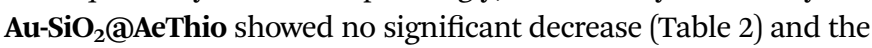
catalyst could be reused at least for five times.

\section{Conclusions}

This paper reports the synthesis of novel heterogeneous catalytic systems (Au-SiO $\mathbf{A}_{2}$ aAeThio) based on gold nanoparticles supported on functionalized silica, chemically modified by a cocondensation reaction involving an alkynylsiloxane further derivatized by a radical thiol-yne coupling. The eventual aminosulfide-branches-substituted silica allowed an efficient in situ generation and concomitant stabilization of low-sized gold nanoparticles without the need of any additional reactant. ${ }^{36}$ The

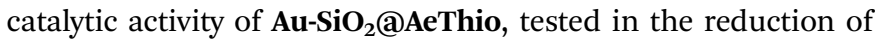
4-NP to 4-AP, proved to be quite notable, with specific kinetic constants ( $\mathrm{ca} .10^{-2} \mathrm{~s}^{-1} \mathrm{mg}_{\text {cat }}{ }^{-1}$ ) comparable to those observed in homogeneous catalytic systems. In addition, the catalysts could be successfully recycled without any significant activity deterioration, an important feature in view of conceivable practical exploitations. This is also the first example of application of radical click chemistry to the field of silica-supported metal nanoparticles, an area that is certainly worth of further investigation for the construction of other interesting catalytic systems.

\section{Acknowledgements}

The authors wish to thank Bologna University for financial support. A. M. thanks the Center for Industrial Research Advanced Applications in Mechanical Engineering and Materials Technology (CIRI-MAM), University of Bologna, for financial support. D. N. acknowledges support by a grant from the Italian MIUR (PRIN 2010-2011-010PFLRJR, "PROxi" project). G. C. and D. B. acknowledge the financial support from Padova University ex-60\% 2012-2015 and Regione Lombardia-INSTM ATLANTE projects. The authors also gratefully thank Dr Alberto Mucchi for AAS measurements and Dr Fabio De Simone (Alfatest s.r.l.) for SEM analyses.

\section{Notes and references}

1 S. Fazzini, M. C. Cassani, B. Ballarin, E. Boanini, J. S. Girardon, A.-S. Mamede, A. Mignani and D. Nanni, J. Phys. Chem. C, 2014, 118, 24538.
2 See the Results and Discussion Section for the amino/ ammonium groups ratios in $\mathbf{S i O}_{2}$ @AeThio.

3 L. Liang and D. Astruc, Coord. Chem. Rev., 2011, 255, 2933.

4 M. Minozzi, A. Monesi, D. Nanni, P. Spagnolo, N. Marchetti and A. Massi, J. Org. Chem., 2011, 76, 450.

5 TEC is the analogous acronym for addition to alkenes.

6 A. Massi and D. Nanni, Org. Biomol. Chem., 2012, 10, 3791.

7 M. Guerrouache, S. Mahouche-Chergui, M. M. Chehimi and B. Carbonnier, Chem. Commun., 2012, 48, 7486.

8 H. Lee, H. Kim, T. J. Choi, H. W. Park and J. Y. Chang, Chem. Commun., 2015, 51, 9805.

9 4-NP is a Clean Water Act priority pollutant, See: Clean Water Act Priority Pollutant List. U.S. Environmental Protection Agency, Code of Federal Regulations, p. 40, CFR 423, Appendix A, 1982, http://www2.epa.gov/eg/toxic-andpriority-pollutants-under-clean-water-act\#priority.

10 R. S. Downing, P. J. Kunkeler and H. van Bekkum, Catal. Today, 1997, 37, 121.

11 G. R. Fulmer, A. J. M. Miller, N. H. Sherden, H. E. Gottlieb, A. Nudelman, B. M. Stoltz, J. E. Bercaw and K. I. Goldberg, Organometallics, 2010, 29, 2176.

12 D. Briggs and M. P. Seah, Practical Surface Analysis: Auger and X-ray Photoelectron Spectroscopy, Wiley, New York, 1990.

13 J. F. Moulder, W. F. Stickle, P. E. Sobol and K. D. Bomben, Handbook of X-ray Photoelectron Spectroscopy, Perkin Elmer Corporation, Eden Prairie, MN, 1992.

14 http://imagej.nih.gov/ij/, accessed May 2015.

15 The amount of $\mathrm{Au}(\mathrm{III})$ precursor was chosen in order to have a nominal total gold amount of $4.7 \mathrm{wt} \%$, in line with previous studies (see ref. 1).

16 We suggest that the low conversion of the TYC reaction is not due to the experimental conditions, but rather mainly to steric and/or electrostatic interactions between the silicaanchored alkyne moieties and cysteamine. In fact, with respect to "free" alkynes, our triple bonds are distributed on the silica surface and, as they react with cysteamine forming the bulky bis-sulfide adduct, they could hinder the analogous reaction of a vicinal alkyne: the latter will be much less accessible to attack by the cysteamine sulfanyl radicals both by steric reasons and by electrostatic interactions between the already anchored cysteamine and the free, approaching one.

17 K. L. Furer, J. Mol. Struct., 1998, 449, 53.

$18 \mathrm{http}: / /$ srdata.nist.gov/xps/.

19 D. Barreca, A. Gasparotto, C. Maccato, E. Tondello and G. Rossetto, Thin Solid Films, 2008, 516, 7393. 
20 S. Fazzini, D. Nanni, B. Ballarin, M. C. Cassani, M. Giorgetti, C. Maccato, A. Trapananti, G. Aquilanti and S. I. Ahmed, J. Phys. Chem. C, 2012, 116, 25434.

21 L. Armelao, D. Barreca, A. Gasparotto, E. Pierangelo, E. Tondello and S. Polizzi, J. Nanosci. Nanotechnol., 2005, 5, 259.

22 D. Barreca, A. Gasparotto, E. Tondello, G. Bruno and M. Losurdo, J. Appl. Phys., 2004, 96, 1655.

23 N. Sahiner, H. Ozay, O. Ozay and N. Aktasc, Appl. Catal., B, 2010, 101, 137.

24 M. Nasrollahzadeha, S. M. Sajadi, A. Rostami-Vartooni, M. Bagherzadeh and R. Safari, J. Mol. Catal. A: Chem., 2015, 400, 22.

25 S. Saha, A. Pal, S. Kundu, S. Basu and T. Pal, Langmuir, 2010, 26, 2885.

26 K. Kuroda, T. Ishida and M. Haruta, J. Mol. Catal. A: Chem., 2009, 298, 7.

27 A. Bilici, B. Ayten and I. Kaya, Synth. Met., 2015, 201, 11.

28 A. M. Khalil, V. Georgiadou, M. Guerrouache, S. MahoucheChergui, C. Dendrinou-Samara, M. M. Chehimi and B. Carbonnier, Polymer, 2015, 77, 218.

29 V. Evangelista, B. Acosta, S. Miridonov, E. Smolentseva, S. Fuentes and A. Simakov, Appl. Catal., B, 2015, 166-167, 518.
30 N. Bingwa and R. Meijboom, J. Mol. Catal. A: Chem., 2015, 396, 1.

31 See: National Institute of Standard and Technology (NIST, http://webbook.nist.gov/) and C. Tosi, UV Atlas of Organic Compounds, 1968, 4, C7/16 for the UV spectrum of the dimethyl-derivative.

32 S. Wada, M. Urano and H. Suzuki, J. Org. Chem., 2002, 67, 8254.

33 Y. Liu, B. Liu, A. Guo, Z. Dong, S. Jin and Y. Lu, Molecules, 2011, 16, 3563.

34 Y. Dai, Y. Li and S. Wang, J. Catal., 2015, 329, 425.

35 H. Kang, M. Kim and K. H. Park, Appl. Catal., A, 2015, 502, 239.

$36 \mathrm{As}$ far as the mechanism of reduction/stabilization is concerned, so far we do not have any indications about the roles of sulfur and nitrogen. However, it has been reported that both moieties can act as reducing/binding agents towards metallic gold and we believe that in our system both mechanisms can occur, see ref. 20 and: E. B. Troughton, C. D. Bain, G. M. Whitesides, R. G. Nuzzo, D. L. Allara and M. D. Porter, Langmuir, 1988, 4, 365; G. J. Ashwell, A. T. Williams, S. A. Barnes, S. L. Chappell, L. J. Phillips, B. J. Robinson, B. UrasinskaWojcik, P. Wierzchowiec, I. R. Gentle and B. J. Wood, J. Phys. Chem. C, 2011, 115, 4200. 